\title{
Psychiatric comorbidities among patients with esophageal cancer in South Korea: a nationwide population-based, longitudinal study
}

\author{
Jaesung Heo ${ }^{1}$, O Kyu Noh $^{1,2,3}$ \\ ${ }^{1}$ Department of Radiation Oncology, ${ }^{2}$ Department of Biomedical Informatics, Ajou University School of Medicine, Suwon, Republic of Korea; \\ ${ }^{3}$ Office of Biostatistics, Ajou Research Institute for Innovative Medicine, Ajou University Medical Center, Suwon, Republic of Korea \\ Contributions: (I) Conception and design: J Heo; (II) Administrative support: OK Noh; (III) Provision of study materials or patients: OK Noh; (IV) \\ Collection and assembly of data: J Heo; (V) Data analysis and interpretation: J Heo; (VI) Manuscript writing: All authors; (VII) Final approval of \\ manuscript: All authors. \\ Correspondence to: O Kyu Noh, MD, PhD. Department of Radiation Oncology, Ajou University School of Medicine, 164 Worldcup-ro, Yeongtong- \\ gu, Suwon 16499, Republic of Korea. Email: okyu.noh@gmail.com.
}

\begin{abstract}
Background: This longitudinal study aimed to analyze the prevalence of psychological distress in esophageal cancer survivors, using claims data in South Korea.

Methods: From January 2010 to December 2014, we identified the four most frequent psychological distress in a nationwide cohort of 8,879 patients diagnosed with esophageal cancer. We analyzed the prevalence and the pattern of psychological distress before and after the initial treatment.

Results: Among esophageal cancer patients, 669 patients (7.5\%) were diagnosed with a psychological distress between 1 year prior to initial treatment and the time of last follow-up. Among them, depression and anxiety had a similar frequency of 237 (35.4\%). The overall frequency of psychological distress peaked within 2 months after the initial treatment. Stress reaction/adjustment disorders showed the highest increase rate after treatment. The rate of patients who had psychological distress was higher among those who underwent surgery as their initial treatment than in those who received radiotherapy [odds ratio $(\mathrm{OR}): 1.39, \mathrm{P}<0.001$ ]. Further, female patients were more likely to be diagnosed with psychological distress compared with male patients (OR: 1.30, $\mathrm{P}<0.001$ ).

Conclusions: Psychological distress in esophageal cancer survivors showed different patterns of prevalence depending on the nature of disease, sex, and initial treatment. Considering individual factors, timely diagnosis and intervention for psychological distress could improve the quality of life for esophageal cancer survivors.
\end{abstract}

Keywords: Cancer survivors; esophageal cancer; depression; anxiety; quality of life

Submitted Dec 09, 2019. Accepted for publication Feb 06, 2020.

doi: $10.21037 /$ jtd.2020.02.55

View this article at: http://dx.doi.org/10.21037/jtd.2020.02.55

\section{Introduction}

Esophageal cancer is the tenth most common cancer worldwide, with approximately 455,800 new cases diagnosed in 2012, and the incidence has been increasing rapidly (1). In South Korea, esophageal cancer accounted for $1.1 \%(2,413)$ of all newly diagnosed cancers in 2014 ( $\mathrm{N}=217,057)$ (2). Esophageal cancer has a relatively poor prognosis, with an overall 5 -year survival rate of less than $10 \%$, owing to a lack of initial symptoms and delayed diagnosis (3). Psychological distress is highly prevalent among cancer survivors because of cancer diagnosis, treatment related side effects, and fear of prognosis after treatment (4). Also, psychiatric comorbidities are higher in esophageal cancer survivors than that in the general population (5). Notably, patients with esophageal cancer showed a higher risk of suicide with peaked risk within the first 3 months after diagnosis (6). These psychological problems can result in poor adherence 
Table 1 Patient characteristics between groups according to main treatment $(\mathrm{N}=8,879)$

\begin{tabular}{lccc}
\hline \multirow{2}{*}{$\begin{array}{l}\text { Patient } \\
\text { characteristics }\end{array}$} & $\begin{array}{c}\text { Main treatment, No. of patients }(\%) \\
(\mathrm{N}=4,883)\end{array}$ & \multicolumn{1}{c}{$\begin{array}{c}\text { Surgery } \\
(\mathrm{N}=3,996)\end{array}$} & \\
\cline { 2 - 3 } & & & 0.534 \\
Sex & $4,532(92.8)$ & $3,694(92.4)$ & \\
Male & $351(7.2)$ & $302(7.6)$ & \\
Female & & & $<0.001$ \\
Age, year & $66.4 \pm 9.7$ & $64.1 \pm 8.6$ & \\
Mean \pm SD & & & $<0.001$ \\
Mental disorder & $317(6.5)$ & $352(8.8)$ & \\
Yes & $4,566(93.5)$ & $3,644(91.2)$ & \\
No & &
\end{tabular}

to treatment and could increase mortality in cancer survivors (7).

In addition to other clinical factors such as tumor stage and prognosis, invasive treatment could be a risk factor for psychological distress (8). Esophagectomy for resectable disease is an extensive surgical procedure and is associated with severe side effects (9). Radiation therapy (RT) may also have side effects such as esophagitis, weight loss, fatigue, and anorexia (10). These treatment-related toxicities could have significant negative impact on the psychological status of esophageal cancer survivors (11).

Information on esophageal cancer survivors currently treated for psychological distress in South Korea is limited because of the small sample size and cross-sectional design of previous studies. Thus, this study aimed to investigate the prevalence of psychological distress among esophageal cancer survivors in Korea, using claims data.

\section{Methods}

The National Health Insurance (NHI) system is the public medical insurance system of South Korea; it covers almost $98 \%$ of the total population (12). The Health Insurance Review and Assessment Service (HIRA) generates data from reimbursement claims for healthcare services given during hospital visits or inpatient admissions. Data include general patient information, healthcare services, diagnosis, outpatient prescription, drug information, and provider information. This study was approved by the institutional review board (IRB) of Ajou University Hospital (IRB No.
AJIRB-MED-EXP-16-494). The informed consent was exempted by the board.

In this study, we used the nationwide cohort data of patients diagnosed with esophageal cancer between January 1, 2010 and December 31, 2014. We included those with esophageal cancer classified as category C15 according to the Korean Classification of Disease, sixth edition, which is based on International Classification of Diseases (ICD) 10th Revision and excluded patients aged less than 19 years old. We also used initial treatment procedure codes such as surgery or RT. Information on psychological distress was obtained from the HIRA database based on the primary diagnostic code. Because we aimed to study the patterns of newly emerging psychiatric conditions before and after the start of cancer treatment, we excluded individuals diagnosed with pre-existing mental disorders 1 year or more before the initiation of cancer therapies.

We analyzed the prevalence of psychiatric distress and its longitudinal pattern according to time sequence. Descriptive statistics were employed to estimate the frequency of psychological distress based on the diagnostic date of the psychological distress. Differences in covariate distribution among groups were determined using chi-square test for categorical variables and $t$-test for continuous variables. A two-sided $\mathrm{P}$ value of less than 0.05 was considered statistically significant. Odds ratio (OR) as an estimate of the relative risk for psychological distress associated with clinical factors and $95 \%$ confidence intervals (CI) were obtained using multivariate logistic regression. All statistical analyses were performed with R 3.0.2. (R Core Team, 2016, Vienna, Austria).

\section{Results}

In total, 8,879 esophageal cancer survivors were identified. Of them, 8,226 (92.6\%) were men, and the median age at diagnosis was 66 years. We compared the age, sex and psychological distress between surgery and RT as main treatment (Table 1). The mean age of the RT group is significantly higher than that of the surgery group. The rate of psychological distress was higher in the surgery group than in the RT group. The frequency of psychological distress among esophageal cancer survivors is shown in Table 2. In total, $669(7.5 \%)$ survivors developed a psychological distress, with depression and anxiety having a similar frequency of 237 (35.4\%). Substance abuse was confirmed in $130(19.4 \%)$ patients; of them, 121 and 9 were diagnosed 
Table 2 The frequency of mental disorders in patients with esophageal cancer $(\mathrm{N}=8,879)$

\begin{tabular}{|c|c|c|c|c|c|c|}
\hline \multirow{3}{*}{ Age (year) } & \multicolumn{6}{|c|}{ No. of patients (\%) } \\
\hline & \multirow[b]{2}{*}{ Esophageal cancer } & \multirow[b]{2}{*}{ Total mental disorders } & \multicolumn{4}{|c|}{ Each mental disorder } \\
\hline & & & Substance abuse & Depressive disorder & Anxiety disorder & $\begin{array}{c}\text { Stress/adjustment } \\
\text { disorder }\end{array}$ \\
\hline 19-39 & 25 & $2(8.0)$ & $1(4.0)$ & $1(4.0)$ & 0 & 0 \\
\hline $60-69$ & 3,212 & $259(8.1)$ & $50(1.6)$ & $85(2.6)$ & $93(2.9)$ & $31(0.1)$ \\
\hline $70-99$ & 3,203 & $220(6.9)$ & $13(0.4)$ & $90(2.8)$ & $95(3.0)$ & $22(0.7)$ \\
\hline Total & 8,879 & $669(7.5)$ & $130(1.5)$ & $237(2.7)$ & $237(2.7)$ & $65(0.7)$ \\
\hline
\end{tabular}

with alcoholism and opiate abuse, respectively.

The pattern of diagnoses for psychological distress is shown in Figure 1. The rate of psychological distress started to increase before the initiation of treatment, peaked around the beginning of treatments, and decreased afterwards. The timing of peak incidences differed between the psychological distress. Depression peaked at 3 months after the start of the cancer therapy, while anxiety peaked just before the start of treatment. The highest rate of increase after treatment was noted for stress reaction/adjustment disorder. Unlike the other mental disorders, substance abuse peaked at 9 months before initial treatment.

With respect to patterns of psychological distress before and after the start of cancer therapies, 257 (38.4\%) and 412 (61.6\%) patients were diagnosed with psychological distress before and after the start of treatment, respectively (Figure 2). Anxiety was the most common disorder reported before the start of treatment (38.5\%), followed by substance abuse (31.1\%). Meanwhile, depression was the most frequent disorder after the start of treatment (42.2\%). With respect to patterns of diagnoses for psychological distress between male and female patients (Figure 3), the rate of substancedependent disorders were higher in men, while anxiety and depression were higher in women. The frequency density of psychological distress tended to fluctuate more in females than males (Figure 4).

The risk of psychological distress tended to be higher in female patients compared to that in male patients (OR: 1.31, 95\% CI: 1.00-1.73, $\mathrm{P}=0.053$ ) (Table 3). The primary treatment modality for esophageal cancer was a significant predictor for the occurrence of psychological distress. The OR for being diagnosed with psychological distress was 1.39 for the surgery group (comparison, RT group; 95\% CI: 1.19-1.63, $\mathrm{P}<0.001$ ).

\section{Discussion}

The treatment for esophageal cancer could induce emotional stress, which in turn can negatively influence disease prognosis (13). The prevalence of psychological distress among cancer survivors also change significantly during the different periods of the malignancy $(14,15)$. In this study, we investigated the prevalence of psychological distress among esophageal cancer survivors and also analyzed changes in the frequency of psychological distress starting from 1 year before treatment, using a nationwide claims data. Of the 8,879 esophageal cancer survivors identified, 669 (7.5\%) developed psychological distress. Anxiety and depression were the most common psychological distress (35.4\%), and its distribution was time dependent.

Patients with esophageal cancer can experience significant psychological distress because of fears from upcoming treatment and treatment-related side effects as well as recurrence or progression. Before treatment, distress can be due to undergoing diagnostic workup for a suspicious malignancy and fear of radical treatment (15). Therefore, pretreatment assessment could help screen for patients who are at risk of psychological distress according to clinicodemographic factors such as sex, age, and initial treatment (16). However, despite several guidelines recommending assessment for mental status among cancer patients, most medical institutions may not screen cancer patients during treatment (17). 


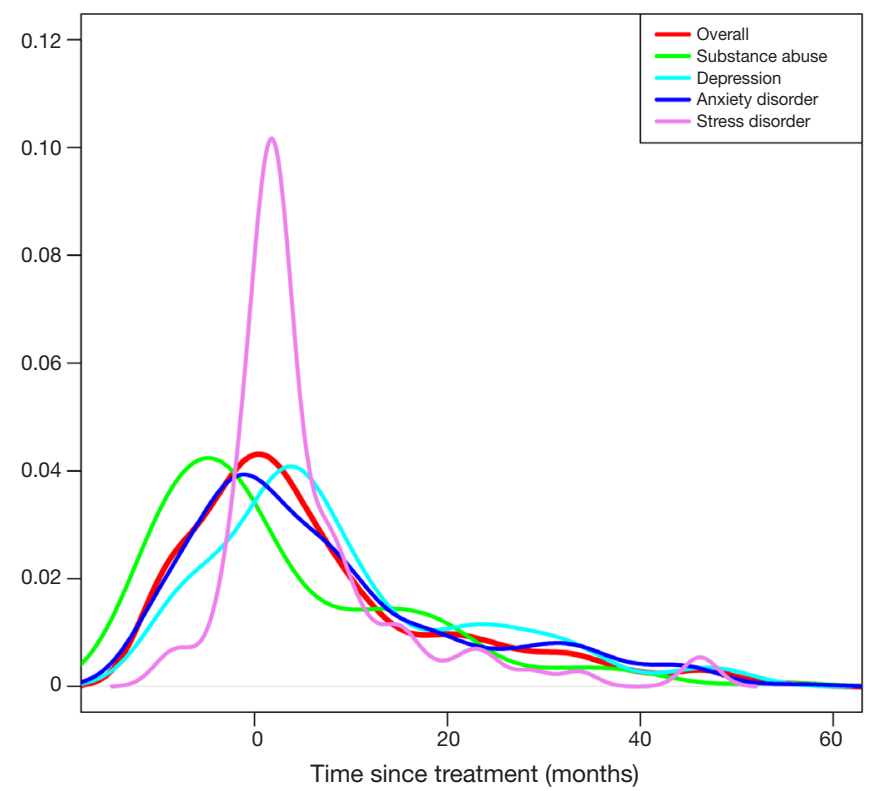

Figure 1 Frequency density of psychological distress in esophageal cancer survivors (the area under density curve for each psychological distress is 1).

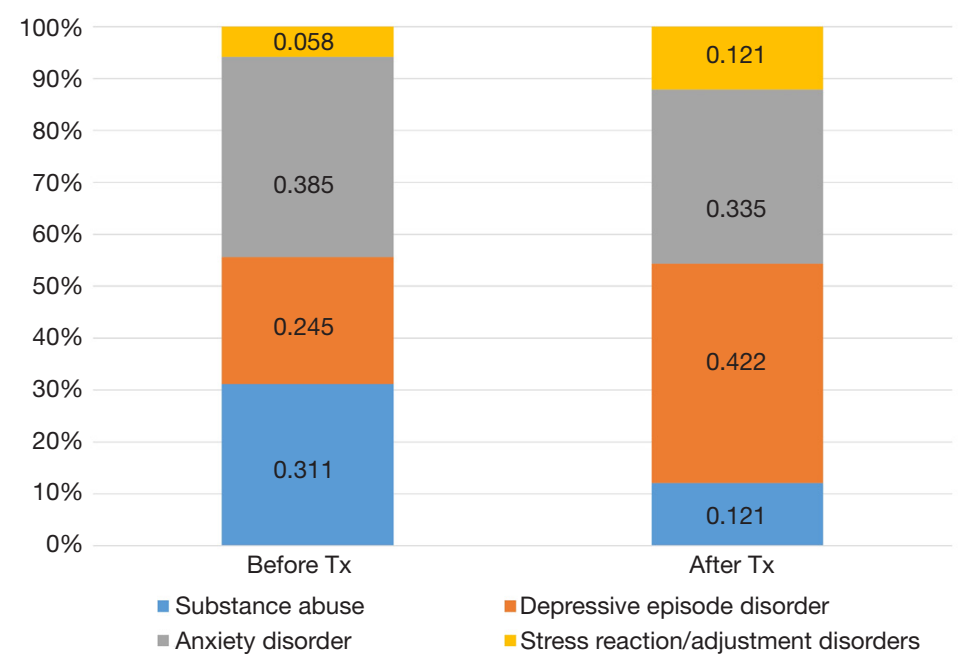

Figure 2 Psychological distress before and after treatment.

In this study, the incidence of psychological distress peaked within 2 months of primary treatment and then gradually decreased (Figure 1). Esophageal cancer survivors are at higher risk of suicide within the first 3 months of diagnosis compared with the cancer-free population (6). In breast cancer survivors, the overall frequency of psychological distress also peaked within 1 month after the cancer diagnosis (18). On the longitudinal approach, esophageal cancer survivors experience distress from the time of diagnosis throughout the first year, and depression increased up to 4 years later (19). In a 20-year follow-up study of cancer survivors, the prevalence of psychological distress decreased and was at a similar level as those with general population (20). Therefore, in addition to the crosssectional approach, these findings support the importance of longitudinal investigations to identify and monitor mental 


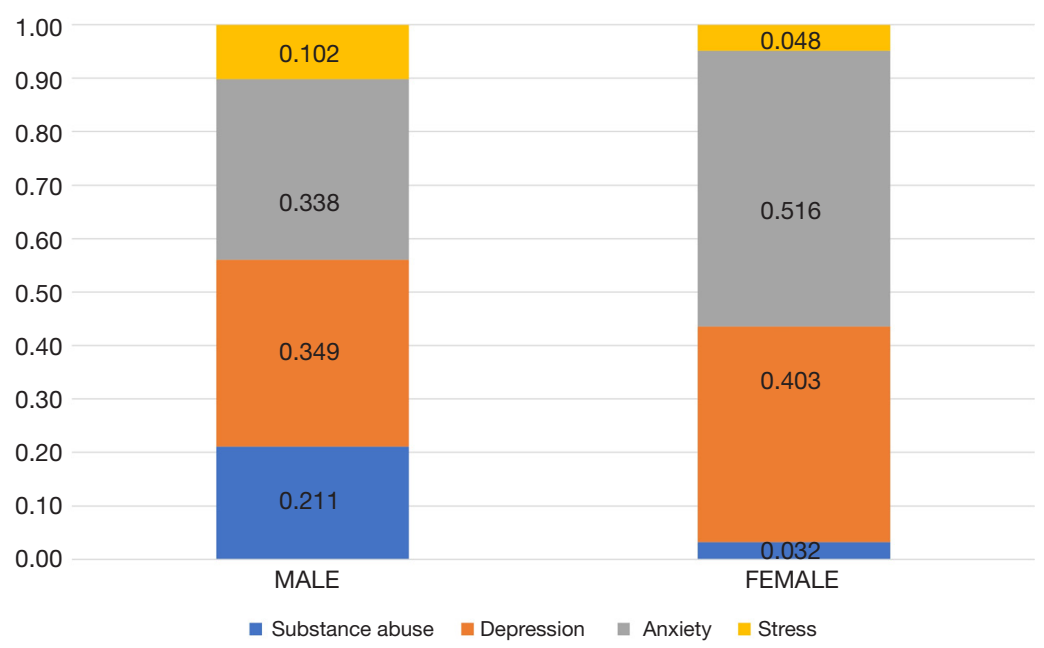

Figure 3 Distribution of psychological distress according to sex.

distress in esophageal cancer survivors.

Each psychological distress showed a different timedependent pattern of manifestation. Before the treatment, anxiety was the highest and then decreased after treatment. In the pre-treatment phase, patients may develop anxiety while anticipating treatment (21). Meanwhile, depression was highly common after treatment, and this may be due to treatment-related acute toxicity. The presence of depression in the post-treatment period could result in non-compliance with adjuvant treatments (22). Compared with other psychological distress, the frequency of stress/adjustment disorders increased rapidly after treatment. In longitudinal studies of cancer survivors, stress reaction/adjustment disorder showed the highest rate of increase after cancer diagnosis $(14,18)$. Therefore, providing personalized intervention based on the time-dependent patterns of each psychological distress could help improve the quality of life for esophageal cancer survivors.

The percentage of substance abuse was higher in esophageal cancer survivors than in the general population ( $1.5 \%$ vs. $0.1 \%$ ) (Table 1$)$. Moreover, substance abuse was higher in esophageal cancer survivors than in survivors of other cancers $(17.5 \%$ vs. $<1 \%)(14,18)$, with alcoholism being the most common form $(90 \%)$. Notably, alcohol consumption has been demonstrated to be a major risk factor for developing esophageal cancer, particularly esophageal squamous cell carcinoma (23). Also, pretreatment alcohol consumption could affect the posttreatment prognosis of esophageal cancer (24). Therefore, substance abuse such as alcoholism should also be managed after treatment to improve the prognosis for esophageal cancer. The finding can be confusing that the peak incidence of substance abuse showed at nine months before initiating cancer treatment. However, this finding may be the result of the incidental and sequential diagnosis of alcoholism and esophageal cancer. While continuing the management for alcohol addiction, esophageal cancer can be detected. Vice versa, before the definite cancer diagnosis, alcoholism can be diagnosed at the time of initial esophageal symptoms.

In this study, women showed higher risk for psychological distress (OR: 1.31), consistent with the findings that female cancer survivors are more at risk for emotional disorders. In a previous study of esophageal cancer patients, women were more likely to report anxiety prior to treatment than men (21). The incidence of psychological distress sharply changed before and after treatment in women. Further, the risk of emotional disorders was significantly higher in the surgery group (HR: $1.33, \mathrm{P}<0.001$ ), which is believed to be related to distress before invasive treatment and the side effects caused by treatment. However, the RT group also showed a high prevalence of psychiatric conditions, which could be a barrier to receive appropriate surgical care. The RT group was older patients and had poor general condition compared to the surgery group (Table 1). These findings suggest that patients perceive RT to be relatively less aggressive treatment than surgery (25). Therefore, it is important to evaluate the risk of psychological distress in cancer survivors, with consideration of clinical factors such as sex and treatment.

This study had some limitations. First, psychological 

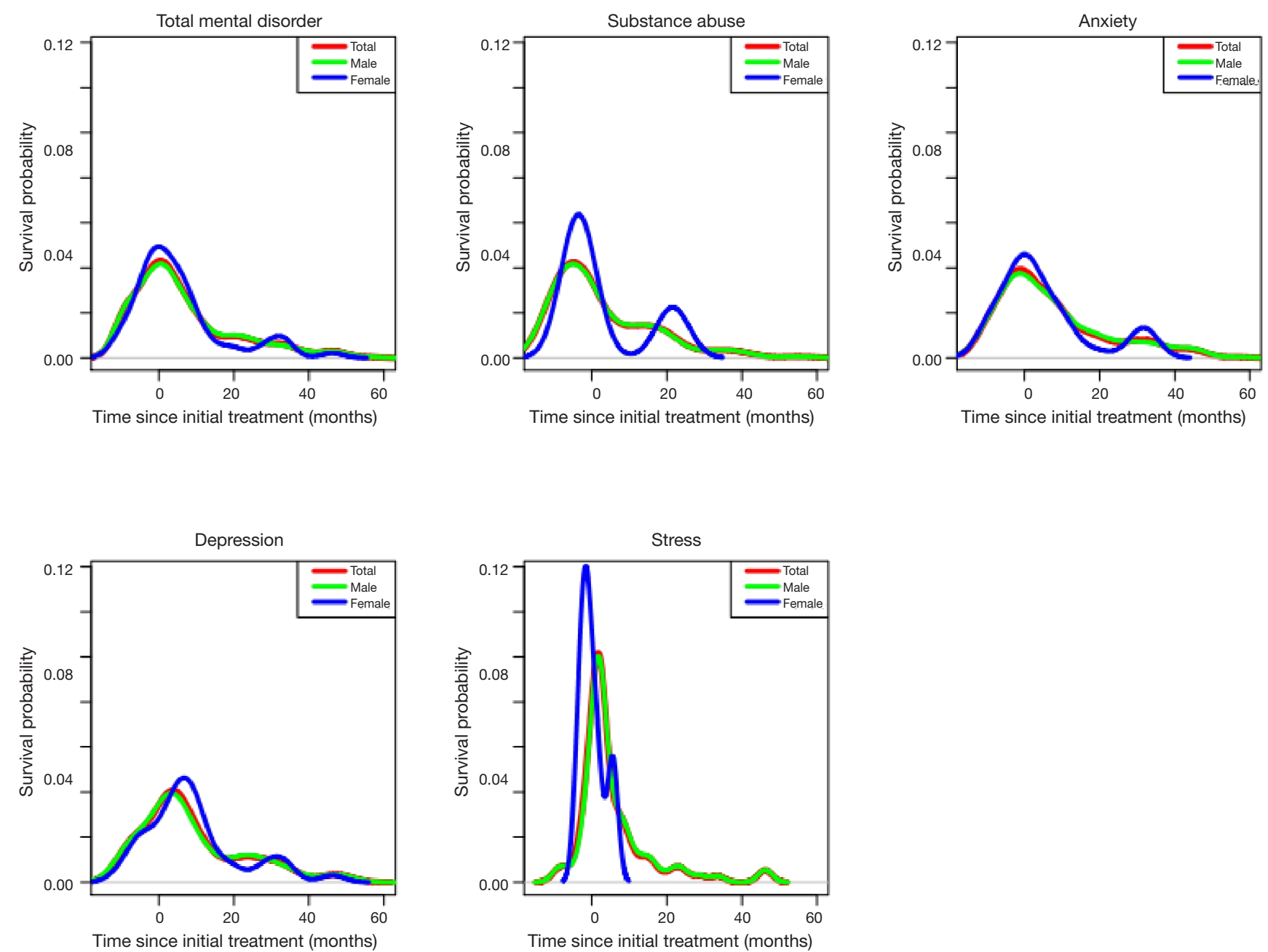

Figure 4 Frequency density of psychological distress in esophageal cancer survivors according to sex (the area under density curve for each psychological distress is 1$)$.

Table 3 Risk factors associated with mental disorders in multivariable analysis $(\mathrm{N}=8,879)$

\begin{tabular}{lcc}
\hline Variable & Odds ratio (95\% confidential interval) & P value \\
\hline Sex & 1 & 0.053 \\
Male & $1.31(1.00-1.73)$ & \\
Female & 1 & 0.212 \\
Age & $1.11(0.94-1.30)$ & \\
$\geq 66$ & & $<0.001$ \\
$<66$ & 1 & \\
Main treatment & $1.39(1.19-1.63)$ & \\
Radiotherapy & & \\
Surgery & & \\
\hline
\end{tabular}

distress was identified based on claims data from the HIRA. Clinical data (stage, histologic type), social data (educational level, marital status) were not available. Notably, the prognosis of esophageal cancer differs according to its histologic features (squamous carcinoma $v s$. adenocarcinoma). Second, the disease codes on claim data using in this study were not generated for the research. The ICD codes for psychological distress may have lesser diagnostic accuracy than those obtained from structured clinical data. However, the prescription for psychotic drugs is highly regulated, and thus mental illnesses are coded cautiously and it could lessen the issue of the accuracy of the diagnosis codes. Because the claim data does not include all the patients with mental illness, the results of this study 
should be interpreted cautiously. Third, we focused on time sequence and analyzed the pattern of prevalence and risk factor during the first diagnosis of psychological distress. Because a cancer survivor could have several psychiatric comorbidities, future studies should consider changes in the mental health status over time.

In conclusion, esophageal cancer patients experience psychological distress before, during, and after the treatment. The psychological distress showed different patterns of prevalence depending on the nature of disease and time sequence. Timely diagnosis and early intervention with a referral system are essential for the management of psychological distress. In particular, given that the risk for psychological distress is higher in women and among those who undergo surgery as primary treatment, individualized preventive measures and clinical intervention are needed.

\section{Acknowledgments}

Funding: This research was supported by the Bio \& Medical Technology Development Program of the National Research Foundation (NRF) funded by the Korean government (MSIT) (No. 2020M3A9E8024907).

\section{Footnote}

Conflicts of Interest: All authors have completed the ICMJE uniform disclosure form (available at http://dx.doi. org/10.21037/jtd.2020.02.55). The authors have no conflicts of interest to declare.

Ethical Statement: The authors are accountable for all aspects of the work in ensuring that questions related to the accuracy or integrity of any part of the work are appropriately investigated and resolved. This study was approved by the institutional review board (IRB) of Ajou University Hospital (IRB No. AJIRB-MED-EXP-16-494). The informed consent was exempted by the board.

Open Access Statement: This is an Open Access article distributed in accordance with the Creative Commons Attribution-NonCommercial-NoDerivs 4.0 International License (CC BY-NC-ND 4.0), which permits the noncommercial replication and distribution of the article with the strict proviso that no changes or edits are made and the original work is properly cited (including links to both the formal publication through the relevant DOI and the license). See: https://creativecommons.org/licenses/by-nc-nd/4.0/.

\section{References}

1. Lagergren J, Lagergren P. Recent developments in esophageal adenocarcinoma. CA Cancer J Clin 2013;63:232-48.

2. Jung KW, Won YJ, Oh CM, et al. Cancer Statistics in Korea: Incidence, Mortality, Survival, and Prevalence in 2014. Cancer Res Treat 2017;49:292-305.

3. Lagergren J, Mattsson F. Diverging trends in recent population-based survival rates in oesophageal and gastric cancer. PLoS One 2012;7:e41352.

4. Mayr M, Schmid RM. Pancreatic cancer and depression: myth and truth. BMC Cancer 2010;10:569.

5. Wikman A, Ljung R, Johar A, et al. Psychiatric morbidity and survival after surgery for esophageal cancer: a population-based cohort study. J Clin Oncol 2015;33:448-54.

6. Papadopoulos FC. Suicide and cardiovascular death after a cancer diagnosis. N Engl J Med 2012;367:276-7; author reply 277.

7. Watson M, Haviland JS, Greer S, et al. Influence of psychological response on survival in breast cancer: a population-based cohort study. Lancet 1999;354:1331-6.

8. Vodermaier A, Linden W, MacKenzie R, et al. Disease stage predicts post-diagnosis anxiety and depression only in some types of cancer. Br J Cancer 2011;105:1814-7.

9. Derogar M, Orsini N, Sadr-Azodi O, et al. Influence of major postoperative complications on health-related quality of life among long-term survivors of esophageal cancer surgery. J Clin Oncol 2012;30:1615-9.

10. Shridhar R, Almhanna K, Meredith KL, et al. Radiation therapy and esophageal cancer. Cancer Control 2013;20:97-110.

11. Hellstadius Y, Lagergren P, Lagergren J, et al. Aspects of emotional functioning following oesophageal cancer surgery in a population-based cohort study. Psychooncology 2015;24:47-53.

12. Kim JA, Yoon S, Kim LY, et al. Towards Actualizing the Value Potential of Korea Health Insurance Review and Assessment (HIRA) Data as a Resource for Health Research: Strengths, Limitations, Applications, and Strategies for Optimal Use of HIRA Data. J Korean Med Sci 2017;32:718-28.

13. Satin JR, Linden W, Phillips MJ. Depression as a predictor of disease progression and mortality in cancer patients: a meta-analysis. Cancer 2009;115:5349-61.

14. Heo J, Chun M, Oh YT, et al. Psychiatric comorbidities among ovarian cancer survivors in South Korea: A 
nationwide population-based, longitudinal study.

Psychooncology 2018;27:1021-6.

15. Lu D, Andersson TM, Fall K, et al. Clinical Diagnosis of Mental Disorders Immediately Before and After Cancer Diagnosis: A Nationwide Matched Cohort Study in Sweden. JAMA Oncol 2016;2:1188-96.

16. Costello EJ. Early Detection and Prevention of Mental Health Problems: Developmental Epidemiology and Systems of Support. J Clin Child Adolesc Psychol 2016;45:710-7.

17. Jacobs M, Anderegg MC, Schoorlemmer A, et al. Patients with oesophageal cancer report elevated distress and problems yet do not have an explicit wish for referral prior to receiving their medical treatment plan. Psychooncology 2017;26:452-60.

18. Heo J, Chun M, Oh YT, et al. Psychiatric comorbidities among breast cancer survivors in South Korea: a nationwide population-based study. Breast Cancer Res Treat 2017;162:151-8.

19. Dempster M, McCorry NK, Brennan E, et al. Psychological distress among survivors of esophageal cancer: the role of illness cognitions and coping. Dis Esophagus 2012;25:222-7.

Cite this article as: Heo J, Noh OK. Psychiatric comorbidities among patients with esophageal cancer in South Korea: a nationwide population-based, longitudinal study. J Thorac Dis 2020;12(4):1312-1319. doi: 10.21037/jtd.2020.02.55
20. Zhao G, Okoro CA, Li J, et al. Current depression among adult cancer survivors: findings from the 2010 Behavioral Risk Factor Surveillance System. Cancer Epidemiol 2014;38:757-64.

21. Hellstadius Y, Lagergren J, Zylstra J, et al. Prevalence and predictors of anxiety and depression among esophageal cancer patients prior to surgery. Dis Esophagus 2016;29:1128-34.

22. Jacobsen PB. Screening for psychological distress in cancer patients: challenges and opportunities. J Clin Oncol 2007;25:4526-7.

23. Islami F, Fedirko V, Tramacere I, et al. Alcohol drinking and esophageal squamous cell carcinoma with focus on light-drinkers and never-smokers: a systematic review and meta-analysis. Int J Cancer 2011;129:2473-84.

24. Ma Q, Liu W, Jia R, et al. Alcohol and survival in ESCC: Prediagnosis alcohol consumption and postoperative survival in lymph node-negative esophageal carcinoma patients. Oncotarget 2016;7:38857-63.

25. Steyerberg EW, Neville B, Weeks JC, et al. Referral patterns, treatment choices, and outcomes in locoregional esophageal cancer: a population-based analysis of elderly patients. J Clin Oncol 2007;25:2389-96. 